\title{
SOLITON RESONANCES FOR MKP-II
}

\author{
Jyh-Hao Lee* and Oktay K. Pashaev ${ }^{\dagger}$ \\ * Institute of Mathematics, Academia Sinica, Taipei, Taiwan \\ $\dagger$ Department of Mathematics, Izmir Institute of Technology, \\ Urla-Izmir, 35430, Turkey
}

July 28,2018

\begin{abstract}
Using the second flow - the Derivative Reaction-Diffusion system, and the third one of the dissipative $\mathrm{SL}(2, \mathrm{R})$ Kaup-Newell hierarchy, we show that the product of two functions, satisfying those systems is a solution of the modified Kadomtsev-Petviashvili equation in $2+1$ dimension with negative dispersion (MKP-II). We construct Hirota's bilinear representation for both flows and combine them together as the bilinear system for MKP-II. Using this bilinear form we find one and two soliton solutions for the MKP-II. For special values of parameters our solution shows resonance behaviour with creation of four virtual solitons. Our approach allows one to interpret the resonance soliton as a composite object of two dissipative solitons in $1+1$ dimensions.
\end{abstract}

\section{Introduction}

The gauge theoretical formulation of the low dimensional gravity models, like the Jackiw-Teitelboim model [1, is based on the Cartan-Einstein vielbein or the moving frame method. In terms of these variables in $1+1$ dimensions one deals with so called BF gauge theory and the zero curvature equations of motion, providing a link with soliton equations [2] But in these variables, as the "square root" of the pseudo-Riemannian metric, the soliton equations have dissipative form, this is why we called them the dissipative solitons or dissipatons [2]. The dissipative version of the Nonlinear Schrodinger equation with a rich resonance dynamics [3] is a couple of nonlinear heat and anti-heat equations, which we called the Reaction-Diffusion system (RD) 2], 3. The dissipaton of that system is the pair of two real functions, one of which is exponentially growing, and another decaying, in space and time. But the product of these two functions has the perfect soliton form. As recently realized [4, if dissipatons of $\mathrm{RD}$ evolves with additional time variable according to the next member after RD of SL(2,R) AKNS hierarchy, with cubic dispersion, then this product can be considered as a soliton of 
$2+1$ dimensional Kadomtsev-Petviashvili equation with negative dispersion (KP-II). This way the resonance behaviour of KP-II solitons was found in terms of dissipatons of the $1+1$ dimensional models. Moreover, in this approach, the novel two resonance soliton of KP-II with four virtual solitons was constructed and interpreted as the degenerate four soliton solution [4].

From another site, as was shown before [6], dissipative version of the Derivative nonlinear Schrodinger equations (DNLS) also admits dissipative soliton solutions with resonance interaction [8]. Moreover, these resonances show the chirality properties, propagating only in one direction. In the present paper, following the strategy of paper [4, by the recursion operator of the Kaup-Newell (KN) hierarchy, first we construct the next dissipative system of the hierarchy with cubic dispersion. Then, using these two members of $\mathrm{SL}(2, \mathrm{R})$ Kaup-Newell hierarchy, we show that the product of dissipaton functions is solution of the modified Kadomtsev-Petviashvili equation with negative dispersion (MKP-II) (Section 2). In Section 3 bilinearization of the two flows allows us to find bilinear form for MKP-II. Chiral resonance dissipatons of DRD and their geometrical meaning are considered in Section 4. One and two soliton solutions of MKP-II are constructed in Section 5. We show the resonance character of these solitons interaction and the chirality property posing restriction on the soliton collision angles. In conclusion we discuss main results of this paper.

\section{MKP-II from Kaup-Newell Hierarchy}

The KN hierarchy has the form 5 ]

$$
\left(\begin{array}{c}
q_{t_{n}} \\
r_{t_{n}}
\end{array}\right)=J L^{n}\left(\begin{array}{c}
q \\
r
\end{array}\right)
$$

where the operator

$$
\left(\begin{array}{ll}
0 & \partial \\
\partial & 0
\end{array}\right)
$$

is the first symplectic form, while

$$
L=\frac{1}{2}\left(\begin{array}{cc}
-\partial-r \partial^{-1} q \partial & -r \partial^{-1} r \partial \\
-q \partial^{-1} q \partial & \partial-q \partial^{-1} r \partial
\end{array}\right)
$$

is the recursion operator of the hierarchy, and $\partial \equiv \partial / \partial x$. The second flow of the hierarchy is the system

$$
\begin{gathered}
q_{t_{2}}=\frac{1}{2}\left[q_{x x}+\left(q^{2} r\right)_{x}\right], \\
r_{t_{2}}=\frac{1}{2}\left[-r_{x x}+\left(r^{2} q\right)_{x}\right],
\end{gathered}
$$


while the third one is

$$
\begin{aligned}
& q_{t_{3}}=-\frac{1}{4}\left[q_{x x}+3 r q q_{x}+\frac{3}{2}\left(r^{2} q^{2}\right) q\right]_{x}, \\
& r_{t_{3}}=-\frac{1}{4}\left[r_{x x}-3 r q r_{x}+\frac{3}{2}\left(r^{2} q^{2}\right) r\right]_{x},
\end{aligned}
$$

For the $\mathrm{SL}(2, \mathrm{R})$ case of $\mathrm{KN}$ hierarchy we have real time variables $t_{2}, t_{3}$ which we denote as $y \equiv t_{2} / 2$, and $t \equiv-t_{3} / 4$. In this case functions $q$ and $r$ are real, and we denote them as

$$
e^{+} \equiv q, \quad e^{-} \equiv-r
$$

Then we have the DRD system [6]

$$
\begin{gathered}
e_{y}^{+}=e_{x x}^{+}-\left(e^{+} e^{-} e^{+}\right)_{x} \\
e_{y}^{-}=-e_{x x}^{-}-\left(e^{+} e^{-} e^{-}\right)_{x}
\end{gathered}
$$

and

$$
\begin{aligned}
& e_{t}^{+}=e_{x x x}^{+}-3\left(e^{+} e^{-} e_{x}^{+}\right)_{x}+\frac{3}{2}\left(\left(e^{+} e^{-}\right)^{2} e^{+}\right)_{x}, \\
& e_{t}^{-}=e_{x x x}^{-}+3\left(e^{+} e^{-} e_{x}^{-}\right)_{x}+\frac{3}{2}\left(\left(e^{+} e^{-}\right)^{2} e^{-}\right)_{x},
\end{aligned}
$$

Now we consider the pair of functions of three variables $e^{+}(x, y, t)$ and $e^{-}(x, y, t)$ satisfying the systems $(2.7)$ and (2.8). These systems are compatible since they belong to the same hierarchy for different times. This can be also checked directly from compatibility condition $e_{t y}^{ \pm}=e_{y t}^{ \pm}$by using following conservation laws for Eqs.(2.7) and (2.8) respectively

$$
\begin{gathered}
\left(e^{+} e^{-}\right)_{y}=\left[\left(e_{x}^{+} e^{-}-e^{+} e_{x}^{-}\right)-\frac{3}{2}\left(e^{+} e^{-}\right)^{2}\right]_{x} \\
\left(e^{+} e^{-}\right)_{t}=\left[\left(e^{+} e^{-}\right)_{x x}-3\left(e_{x}^{+} e_{x}^{-}\right)+3\left(e^{+} e^{-}\right)\left(e^{+} e_{x}^{-}-e_{x}^{+} e^{-}\right)+\frac{5}{2}\left(e^{+} e^{-}\right)^{3}\right]_{x}
\end{gathered}
$$

Proposition 1. Let the functions $e^{+}(x, y, t)$ and $e^{-}(x, y, t)$, are solutions of the systems (2.7) and (2.8) simultaneously. Then, the function $U(x, y, t) \equiv$ $e^{+} e^{-}$satisfies the modified Kadomtsev-Petviashvili equation (MKP-II)

$$
\left(-4 U_{t}+U_{x x x}-\frac{3}{2} U^{2} U_{x}-3 U_{x} \partial_{x}^{-1} U_{y}\right)_{x}=-3 U_{y y}
$$

or written in another form

$$
\begin{gathered}
-4 U_{t}+U_{x x x}-\frac{3}{2} U^{2} U_{x}-3 U_{x} W=-3 W_{y} \\
W_{x}=U_{y}
\end{gathered}
$$


(The second form appears from the first one by introducing auxiliary variable $W$ according to Eq.(2.11b) and integration in variable $x$ ).

The proof is straightforward. From definition of $U$ and $\operatorname{Eqs}(2.7),(2.9)$ we have

$$
\begin{gathered}
U_{y}=\left[\left(e_{x}^{+} e^{-}-e^{+} e_{x}^{-}\right)-\frac{3}{2} U^{2}\right]_{x} \\
U_{y y}=\left[U_{x x x}-4\left(e_{x}^{+} e_{x}^{-}\right)_{x}-3 U_{x}\left(e_{x}^{+} e^{-}-e^{+} e_{x}^{-}\right)-U\left(e_{x}^{+} e^{-}-e^{+} e_{x}^{-}\right)_{x}-\frac{3}{2}\left(U^{2}\right)_{y}\right]_{x}
\end{gathered}
$$

From another site Eqs.(2.8),(2.9) give

$$
\begin{array}{r}
U_{t}=\left[U_{x x}-3\left(e_{x}^{+} e_{x}^{-}\right)-3 U\left(e_{x}^{+} e^{-}-e^{+} e_{x}^{-}\right)+\frac{5}{2} U^{3}\right]_{x} \\
U_{t x}=\left[U_{x x}-3\left(e_{x}^{+} e_{x}^{-}\right)-3 U\left(e_{x}^{+} e^{-}-e^{+} e_{x}^{-}\right)+\frac{5}{2} U^{3}\right]_{x x}
\end{array}
$$

First we combine Eqs.(2.12) and (2.14) to cancel term $e_{x}^{+} e_{x}^{-}$, then use Eq.(2.12) to exclude $e_{x}^{+} e^{-}-e^{+} e_{x}^{-}$and its derivative, according to

$$
\left(e_{x}^{+} e^{-}-e^{+} e_{x}^{-}\right)_{x}=U_{y}-\frac{3}{2}\left(U^{2}\right)_{x}
$$

and integrating once

$$
\left(e_{x}^{+} e^{-}-e^{+} e_{x}^{-}\right)=\partial_{x}^{-1} U_{y}-\frac{3}{2} U^{2}
$$

As a result we arrive with the MKP-II (2.10)

\section{Bilinear Form for the Second and Third flows}

Now we will construct bilinear representation for systems (2.7) and (2.8) to find solutions of MKP-II according to our Proposition 1. In our paper 6] we applied the Hirota bilinear method to integrate RDR (2.7). Now we will apply the same method to Eq.(2.8) and MKP-II. As was noticed in [6], the standard Hirota substitution as the ratio of two functions doesn't work directly for $e^{+}$and $e^{-}$. (This fact also is related with complicated analytical structure of DNLS [7]). To have the standard Hirota substitution, following [6] we first rewrite the systems $(2.7)$ and (2.8) in terms of new functions $Q^{+}$, $Q^{-}$:

$$
e^{+}=e^{+\int^{x} Q^{+} Q^{-}} Q^{+}, e^{-}=e^{-\int^{x} Q^{+} Q^{-}} Q^{-},
$$

and as result we have the systems

$$
\begin{gathered}
Q_{y}^{+}=Q_{x x}^{+}+Q^{+} Q^{+} Q_{x}^{-}-\frac{1}{2}\left(Q^{+} Q^{-}\right)^{2} Q^{+} \\
Q_{y}^{-}=-Q_{x x}^{-}+Q^{-} Q^{-} Q_{x}^{+}+\frac{1}{2}\left(Q^{+} Q^{-}\right)^{2} Q^{-}
\end{gathered}
$$


and

$$
\begin{aligned}
& Q_{t}^{+}=Q_{x x x}^{+}+3 Q_{x}^{+} Q_{x}^{-} Q^{+}-\frac{3}{2}\left(Q^{+} Q^{-}\right)^{2} Q_{x}^{+}, \\
& Q_{t}^{-}=Q_{x x x}^{-}-3 Q_{x}^{+} Q_{x}^{-} Q^{-}-\frac{3}{2}\left(Q^{+} Q^{-}\right)^{2} Q_{x}^{-},
\end{aligned}
$$

Then, due to the fact that

$$
Q^{+} Q^{-}=e^{+} e^{-}=U
$$

the systems (3.2), (3.3) provide also solution of MKP-II which we can formulate as below.

Proposition 2. Let the functions $Q^{+}(x, y, t)$ and $Q^{-}(x, y, t)$, are solutions of the systems (3.2) and (3.3) simultaneously. Then, the function $U(x, y, t) \equiv Q^{+} Q^{-}$satisfies the modified Kadomtsev-Petviashvili equation (MKP-II) (2.10) or (2.11).

To solve the systems (3.2) and (3.3) we introduce four real functions $g^{+}, g^{-}, f^{+}, f^{-}$according to the formulas

$$
Q^{+}=\frac{g^{+}}{f^{+}}, \quad Q^{-}=\frac{g^{-}}{f^{-}}
$$

or using Eqs.(3.1) and (3.4) for the original variables $e^{+}$and $e^{-}$we have the following substitution

$$
e^{+}=\frac{g^{+} f^{+}}{\left(f^{-}\right)^{2}}, \quad e^{-}=\frac{g^{-} f^{-}}{\left(f^{+}\right)^{2}} .
$$

Then the system (3.2) bilinearizes in the form

$$
\begin{gathered}
\left(D_{y} \mp D_{x}^{2}\right)\left(g^{ \pm} \cdot f^{ \pm}\right)=0, \\
D_{x}^{2}\left(f^{+} \cdot f^{-}\right)+\frac{1}{2} D_{x}\left(g^{+} \cdot g^{-}\right)=0, \\
D_{x}\left(f^{+} \cdot f^{-}\right)-\frac{1}{2} g^{+} g^{-}=0 .
\end{gathered}
$$

In a similar way, for the system (3.3) we have the next bilinear form

$$
\begin{gathered}
\left(D_{t}-D_{x}^{3}\right)\left(g^{ \pm} \cdot f^{ \pm}\right)=0, \\
D_{x}^{2}\left(f^{+} \cdot f^{-}\right)+\frac{1}{2} D_{x}\left(g^{+} \cdot g^{-}\right)=0 \\
D_{x}\left(f^{+} \cdot f^{-}\right)-\frac{1}{2} g^{+} g^{-}=0 .
\end{gathered}
$$

Comparing these two bilinear forms we can see that the second and the third equations in both systems $(3.7),(3.8)$ are of the same form. This is 
why for simultaneous solution of both Eqs.(3.2), (3.3) we have the next bilinear system

$$
\begin{gathered}
\left(D_{y} \mp D_{x}^{2}\right)\left(g^{ \pm} \cdot f^{ \pm}\right)=0, \\
\left(D_{t}-D_{x}^{3}\right)\left(g^{ \pm} \cdot f^{ \pm}\right)=0, \\
D_{x}^{2}\left(f^{+} \cdot f^{-}\right)+\frac{1}{2} D_{x}\left(g^{+} \cdot g^{-}\right)=0, \\
D_{x}\left(f^{+} \cdot f^{-}\right)-\frac{1}{2} g^{+} g^{-}=0 .
\end{gathered}
$$

¿From the last equation we have

$$
U=e^{+} e^{-}=Q^{+} Q^{-}=\frac{g^{+} g^{-}}{f^{+} f^{-}}=2 \frac{D_{x}\left(f^{+} \cdot f^{-}\right)}{f^{+} f^{-}}=2 \frac{f_{x}^{+} f^{-}-f^{+} f_{x}^{-}}{f^{+} f^{-}}
$$

which provides for solution of MKP-II the following formula

$$
U=2\left(\ln \frac{f^{+}}{f^{-}}\right)_{x}
$$

\section{Resonance Solitons of DRD}

First of all we consider DRD (2.7) as an evolution equation where $y=x_{0}=t$ is interpreted as the time variable.

\subsection{Chiral Dissipative Soliton Solution}

For one dissipaton solution we have

$$
g^{ \pm}=e^{\eta^{ \pm}}, \quad f^{ \pm}=1+e^{\phi^{ \pm}} e^{\eta^{+}+\eta^{-}}, e^{\phi^{ \pm}}= \pm \frac{k^{\mp}}{2\left(k^{+}+k^{-}\right)^{2}},
$$

where $\eta^{ \pm}=k^{ \pm} x \pm\left(k^{ \pm}\right)^{2} t+\eta_{0}^{ \pm}$. The regularity requires that we choose conditions $k^{-}>0$ and $k^{+}<0$ so that we have dissipaton

$$
Q^{ \pm}=\frac{e^{ \pm\left(\eta^{+}-\eta^{-}\right) / 2-\alpha_{ \pm} / 2}}{2 \cosh \frac{1}{2}\left(\eta^{+}+\eta^{-}+\alpha_{ \pm}\right)}
$$

with solitonic density

$$
e^{+} e^{-}=Q^{+} Q^{-}=\frac{2 k^{2}}{\sqrt{v^{2}-k^{2}} \cosh k\left(x-v t-x_{0}\right)+v} .
$$

In the last equation we introduced the dissipaton's amplitude and velocity, $k=k^{+}+k^{-}$and $v=k^{-}-k^{+}$correspondingly, in terms of which the above conditions mean that velocity of dissipaton is bounded from below by $k$. It is worth noting that in contrast with dissipatons of RD system [3], in our case no critical value from above for dissipaton's velocity exists. 
For the mass and momentum densities we have

$$
\begin{gathered}
\rho=e^{+} e^{-}=2 \partial_{x} \ln \frac{f^{+}}{f^{-}} \\
p=\frac{1}{2}\left(e^{+} \partial_{x} e^{-}-e^{-} \partial e^{+}+\left(e^{+} e^{-}\right)^{2}\right)=\partial_{x}^{2} \ln f^{+} f^{-},
\end{gathered}
$$

which allows one calculate the corresponding conserved quantities

$$
M=\int_{-\infty}^{+\infty} \rho d x=\left.2 \ln \frac{f^{+}}{f^{-}}\right|_{-\infty} ^{+\infty}, \quad P=\int_{-\infty}^{+\infty} p d x=\left.\partial_{x} \ln f^{+} f^{-}\right|_{-\infty} ^{+\infty}
$$

Then for mass and momentum of a single dissipaton we obtain

$$
M=\ln \left(\frac{v+k}{v-k}\right)^{2}, \quad P=k .
$$

Due to relation $|v|>|k|$ the mass $M$ is positive. Rewriting momentum in the canonical form $k=\mu v$, we find for the effective mass $\mu=\tanh M / 4$.

\subsection{Geometrical Interpretation}

The model has geometrical interpretation of two dimensional pseudo-Riemannian spacetime with constant scalar curvature $R=\Lambda<0$ [2, 3]. This model is known as the Jackiw-Teitelboim gravity [1. It admits gauge theoretical formulation as the BF theory. The gauge potentials are Cartan-Einstein zweibein fields $e_{\mu}^{ \pm}$, so that the metric is $g_{\mu \nu}=\left(e_{\mu}^{+} e_{\nu}^{-}+e_{\nu}^{+} e_{\mu}^{-}\right) / 2$, and the spin-connection $\omega_{\mu},(\mu=0,1)$. Then equations of motion

$$
\begin{gathered}
D_{\mu}^{\mp} e_{\nu}^{ \pm}=D_{\nu}^{\mp} e_{\mu}^{ \pm}, \\
\partial_{\mu} \omega_{\nu}-\partial_{\nu} \omega_{\mu}=-\frac{\Lambda}{4}\left(e_{\mu}^{+} e_{\nu}^{-}-e_{\nu}^{+} e_{\mu}^{-}\right),
\end{gathered}
$$

where $D_{\mu}^{ \pm}=\partial_{\mu} \pm \omega_{\mu}$, have meaning of torsionless and the constant curvature conditions respectively. We fix the gauge freedom and the corresponding evolution by the following conditions on Lagrange multipliers

$$
e_{0}^{ \pm}= \pm\left(\partial_{1} \mp \frac{\Lambda}{4} \mp e^{+} e^{-}\right) e^{ \pm}
$$

and spin connections

$$
\omega_{0}=\frac{\Lambda^{2}}{16}+\frac{\Lambda}{4} e^{+} e^{-}, \quad \omega_{1}=-\frac{\Lambda}{4} .
$$

Then after the identification $(t, x)=\left(x_{0}, x_{1}\right)$ the system (4.8), (4.9) reduces to $\mathrm{DRD}(2.7)$. It is worth noting that in contrast to the RD [3] in our case 
the scalar curvature disappears from equations of motion but is still present in the linear problem. Moreover it has the meaning of the squared spectral parameter $\Lambda=-8 \lambda^{2}$.

The metric tensor component $g_{00}$ in terms of transformed variables (3.1) is given by

$$
g_{00}=e_{0}^{+} e_{0}^{-}=-\left(\partial_{1}-\frac{\Lambda}{4}\right) Q^{+}\left(\partial_{1}+\frac{\Lambda}{4}\right) Q^{-} .
$$

Calculating it for one dissipaton (4.2) we find that event horizon $g_{00}=0$ exists only for the bounded velocity $-k+(-\Lambda / 2)<v<k+(-\Lambda / 2)$ at the distance

$$
x_{H}-v t_{H}-x_{0 H}=\frac{1}{k} \ln \frac{4 k^{2}\left(k-v-\frac{\Lambda}{2}\right)}{(k+v)\left(k+v+\frac{\Lambda}{2}\right)} .
$$

In contrast to the RD 3 dissipaton with two symmetrical event horizons reflecting two directions of motion, we have now only one directional motion and call the corresponding single event horizon as the chiral event horizon.

\section{$5 \quad$ Resonance Solitons of MKP-II}

Now we consider a solution of the system (3.9), giving $2+1$ dimensional solution of MKP-II. For one-soliton solution we have

$$
g^{ \pm}=e^{\eta_{1}^{ \pm}}, f^{ \pm}=1+e^{\phi_{11}^{ \pm}} e^{\eta_{1}^{+}+\eta_{1}^{-}}, e^{\phi_{11}^{ \pm}}= \pm \frac{k_{1}^{\mp}}{2\left(k_{1}^{+}+k_{1}^{-}\right)^{2}}
$$

where, $\eta_{1}^{ \pm}=k_{1}^{ \pm} x \pm\left(k_{1}^{ \pm}\right)^{2} y+\left(k^{ \pm}\right)^{3} t+\eta_{0}^{ \pm}$. The regularity condition requires $k_{1}^{+} \leq 0, k_{1}^{-} \geq 0$. Then we have

$$
U(x, y, t)=\frac{2 k^{2}}{\sqrt{p^{2}-k^{2}} \cosh k\left(x-p y+\frac{k^{2}+3 p^{2}}{4} t-a_{0}\right)+p},
$$

where $k=k_{1}^{+}+k_{1}^{-}, p=k_{1}^{-}-k_{1}^{+}>0$, and bounded from the below parameter $p^{2}>k^{2}$ is positive $p>0$. The geometrical meaning of this parameter is $p^{-1}=\tan \alpha$, where $\alpha$ is the slope of the soliton line. Due to the condition $p>0$, the direction of this line is restricted between $0<\alpha<\pi / 2$. (This is the space analog of the chirality property of dissipaton in $1+1$ dimensions for DNLS [7], when it propagates only in one direction.) The velocity of soliton is two dimensional vector $\mathbf{v}=(\omega,-\omega / p)$, where $\omega=\left(k^{2}+3 p^{2}\right) / 4$, directed at angle $\gamma$ to the soliton line, where $\cos \gamma=1-1 / p^{2}$. When $p=1$, the velocity of soliton is orthogonal to the soliton line.

For two soliton solution we have

$$
g^{ \pm}=e^{\eta_{1}^{ \pm}}+e^{\eta_{2}^{ \pm}}+\alpha_{1}^{ \pm} e^{\eta_{2}^{+}+\eta_{2}^{-}+\eta_{1}^{ \pm}}+\alpha_{2}^{ \pm} e^{\eta_{1}^{+}+\eta_{1}^{-}+\eta_{2}^{ \pm}}
$$




$$
f^{ \pm}=1+\sum_{i, j=1}^{2} e^{\phi_{i j}^{ \pm}} e^{\eta_{i}^{+}+\eta_{j}^{-}}+\beta^{ \pm} e^{\eta_{1}^{+}+\eta_{1}^{-}+\eta_{2}^{+}+\eta_{2}^{-}},
$$

where $\eta_{i}^{ \pm}=k_{i}^{ \pm} x \pm\left(k_{i}^{ \pm}\right)^{2} y+\left(k^{ \pm}\right)^{3} t+\eta_{i 0}^{ \pm}, k_{i j}^{n m} \equiv\left(k_{i}^{n}+k_{j}^{m}\right)$ and

$$
\begin{gathered}
\alpha_{1}^{ \pm}= \pm \frac{1}{2} \frac{k_{2}^{\mp}\left(k_{1}^{ \pm}-k_{2}^{ \pm}\right)^{2}}{\left(k_{22}^{+-}\right)^{2}\left(k_{12}^{ \pm \mp}\right)^{2}}, \quad \alpha_{2}^{ \pm}= \pm \frac{1}{2} \frac{k_{1}^{\mp}\left(k_{1}^{ \pm}-k_{2}^{ \pm}\right)^{2}}{\left(k_{11}^{+-}\right)^{2}\left(k_{21}^{ \pm \mp}\right)^{2}}, \\
\beta^{ \pm}=\frac{\left(k_{1}^{+}-k_{2}^{+}\right)^{2}\left(k_{1}^{-}-k_{2}^{-}\right)^{2}}{4\left(k_{11}^{+-} k_{12}^{+-} k_{21}^{+-} k_{22}^{+-}\right)^{2}} k_{1}^{\mp} k_{2}^{\mp}, \\
e^{\phi_{i i}^{ \pm}}= \pm \frac{k_{i}^{\mp}}{2\left(k_{i i}^{+-}\right)^{2}}, \quad e^{\phi_{i j}^{+}}=\frac{k_{j}^{-}}{2\left(k_{i j}^{+-}\right)^{2}}, \quad e^{\phi_{i j}^{-}}=-\frac{k_{i}^{+}}{2\left(k_{i j}^{+-}\right)^{2}} .
\end{gathered}
$$

The regularity conditions now are the same as for one soliton $k_{i}^{+} \leq 0, k_{i}^{-} \geq 0$. Then this solution describes a collision of two solitons propagating in plane and at some value of parameters creating the resonance states (Fig.1a,b).

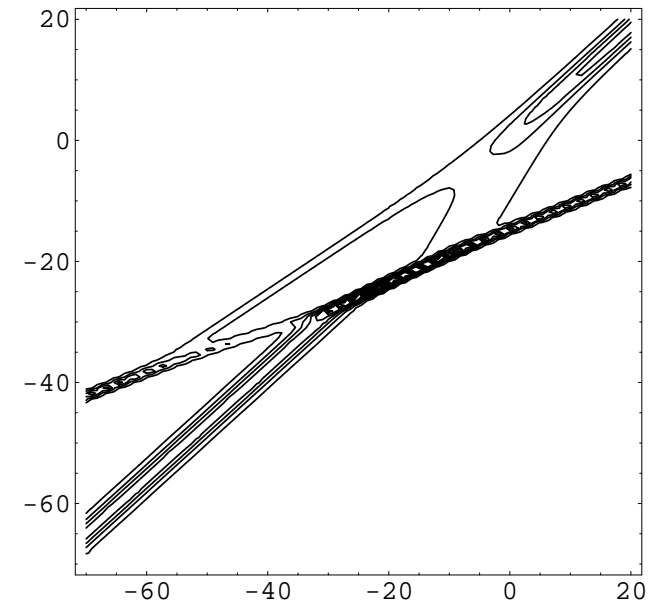

Fig.1a

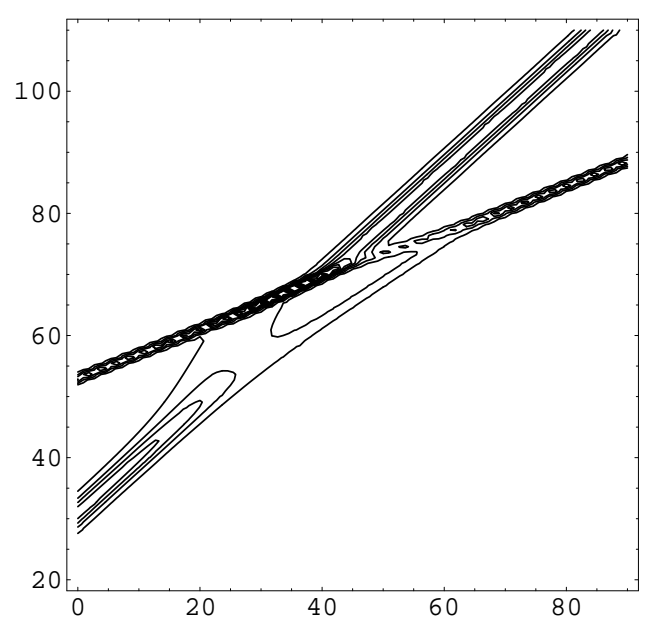

Fig. 1b

\section{Conclusion}

In the present paper we have constructed virtual soliton resonance solutions for MKP-II in terms of dissipative solitons of $1+1$ dimensional equations as the Derivative Reaction-Diffusion equation and its higher member of $\operatorname{SL}(2, \mathrm{R})$ 
Kaup-Newell hierarchy. The difference with the KP-II resonance is in the additional restrictions on solitons angles from regularity conditions.

When this paper has been finished, on the Conference on Nonlinear Physics in Gallipoli, Italy, June-July 2004, Konopelchenko attracted our attention to the relations between MKP equation and $1+1$ dimensional models by the symmetry reduction of $2+1$ dimensional models [9], 10]. But in paper 9] a relation of MKP only with Burgers hierarchy has been established. While paper [10] relates MKP with derivative NLS in the Nakamura-Chen form but not in the Kaup-Newell form. Moreover no results on resonance solitons in those papers are found.

\section{Acknoweledgments}

The authors would like to thanks B. Konopelchenko for useful remarks and Y. Kodama for fruitful discussions. This work was supported partially by Institute of Mathematics, Academia Sinica, Taipei, Taiwan and Izmir Institute of Technology, Izmir, Turkey.

\section{References}

[1] R. Jackiw, in Quantum Theory of Gravity, ed. S. Christensen (Adam Hilger, 1984), p. 403; C. Teitelboim, ibid, p. 327.

[2] L. Martina, O.K. Pashaev and G. Soliani, Class. Quantum Grav. 14 (1997)3179; Phys. Rev. D, 58 (1998) 084025.

[3] O.K. Pashaev and J.-H. Lee, "Resonance Solitons as Black Holes in Madelung Fluid", Modern Physics Letters A, vol. 17, (2002) 16011619 .

[4] O.K. Pashaev and M.L.Y. Francisco, "Degenerate Four Virtual Soliton Resonance for KP-II" (in preparation)

[5] Z. Yan Chaos, Solitons and Fractals, 14 (2002) 45-56.

[6] J.H.-Lee, C.-K. Lin and O.K. Pashaev, "Equivalence relation and Bilinear representation for derivative NLS type equations", in "20 years after NEEDS'79", Proc. of Conference, World Sci. Pub. Singapore (2000)175-181.

[7] J. H.-Lee, "Global solvability of the derivative nonlinear Schrodinger equation", Transactions of the AMS, 314(1), (1989) 107-118.

[8] O.K. Pashaev and J.-H. Lee, "Black Holes and Solitons of quantized dispersionless NLS and DNLS", ANZIAM, vol.44 , (2002) 73-81.

[9] B. Konopelchenko and W. Strampp, J. Math. Phys., vol.33 , (1992) 3676-3686. 
[10] Yi Cheng and Yi-shen Li, "Constraints of the 2+1 dimensional integrable soliton systems", J. Phys. A: Math. Gen., vol.25 , (1992) 419-431. 